\title{
A strong astrophysical constraint on the violation of special relativity by quantum gravity*
}

\author{
T. Jacobson, S. Liberati, D. Mattingly \\ Department of Physics, \\ University of Maryland, College Park, MD 20742-4111, USA.
}

\begin{abstract}
Special relativity asserts that physical phenomena appear the same for all inertially moving observers. This symmetry, called Lorentz symmetry, relates long wavelengths to short ones: if the symmetry is exact it implies that spacetime must look the same at all length scales. Several approaches to quantum gravity, however, suggest that there may be a Lorentz violating microscopic structure of spacetime, for example discreteness [1], non-commutativity [2], or extra dimensions [3]. Here we determine a very strong constraint on a type of Lorentz violation that produces a maximum electron speed less than the speed of light. We use the observation of 100 $\mathrm{MeV}$ synchrotron radiation from the Crab nebula to improve the previous limits by a factor of 40 million, ruling out this type of Lorentz violation, and thereby providing an important constraint on theories of quantum gravity.

To characterize Lorentz violation we adopt the simple framework of deformed dispersion relations. A dispersion relation is the relation between energy $E$ and momentum $p$ for a particle. In relativity, these quantities change under a Lorentz transformation, but the particle dispersion relation $E^{2}=m^{2} c^{2}+p^{2} c^{4}$ is invariant (where $m$ is the particle mass and $c$ is the
\end{abstract}

${ }^{*}$ Previous title: Lorentz violation and Crab synchrotron emission: a new constraint far beyond the Planck scale 
speed of light). Lorentz transformations include both "boosts", which are transformations to a relatively moving frame, and rotations.

We consider dispersion relations that break boost invariance but preserve rotation invariance. (A dispersion relation that is not boost invariant can hold in only one frame. We assume this frame coincides with that of the cosmic microwave background.) In particular for photons and electrons we consider the dispersion relations

$$
\begin{aligned}
& \omega^{2}(k)=k^{2}+\xi \frac{k^{3}}{M}, \\
& E^{2}(p)=m^{2}+p^{2}+\eta \frac{p^{3}}{M},
\end{aligned}
$$

where $\omega$ and $k$ are the photon frequency and wave number, and $E$ and $p$ are the electron energy and momentum. We use units with Planck's constant $\hbar$ and the low energy speed of light $c$ both set to unity (thus the photon energy $\hbar \omega$ is also denoted by $\omega$ ). The energy scale $M=10^{19} \mathrm{GeV}$, which is close to the Planck energy, $M_{\text {Planck }}=1.22 \times 10^{19} \mathrm{GeV}$, is factored out explicitly. We regard these relations as phenomenological, not fundamental. In particular one would expect a whole series of higher order terms in the momentum, but those would be negligible at the energies we consider. Extra lower order terms are already strongly enough constrained by observation so as to be irrelevant for the new constraint considered here.

Dispersion relations with higher order momentum corrections analogous to (11) are familiar from common physical situations like vibrational waves in a crystal or light waves in a refractive medium, where the long wavelength (low momentum) modes travel at a common speed, while the modes whose wavelength is short enough to be sensitive to the microscopic structure of the medium behave differentially. Several approaches to quantum gravity propose a Lorentz violating microscopic structure of spacetime. There is no unique prediction regarding the values of the dimensionless coefficients $\xi$ and $\eta$, but if not suppressed by some other symmetry they would presumably be of order unity if they originate from quantum gravity effects. This is because physics rarely produces dimensionless numbers that differ by many orders of magnitude from unity from theories with only one scale, and the unique energy scale $M$ of quantum gravity has already been factored out in (12).

It is of course possible that other symmetries along with boost symmetry are violated. However, a bound on pure boost violation is also a bound on such multiple symmetry violations, barring an unlikely cancellation of 
different effects. Hence, to keep the analysis simple we assume all of standard physics except boost invariance. In particular we preserve rotation symmetry and electromagnetic gauge invariance, additivity of energy and momentum for multi-particle systems, and energy-momentum conservation in particle interactions.

A consistent dynamical framework that yields modified dispersion and preserves our other assumptions is effective field theory. Effective field theory is very general and can incorporate Lorentz violation arising from a wide range of underlying quantum gravity scenarios, including for example string theory and spacetime discreteness. It has been shown [4] in effective field theory that left and right polarizations of photons have opposite values of $\xi$ in (11), while left and right electron chiralities can have independent $\eta$ values. Polarization dependence of $\xi$ is unimportant for the new constraint described here, although we shall use it at the end when combining our new constraint with previous work. We assume here that $\eta$ is not chirality dependent.

Perhaps surprisingly, Planck energies are not needed to get constraints on $\xi$ and $\eta$ of order unity or even less. For example, the group velocities $d \omega / d k$ and $d E / d p$ for photons and electrons satisfying equations (12) depend on these parameters and on the particle energy. Over long propagation distances this could produce observable arrival time differences for simultaneously emitted photons of different energies [5, 6]. Arrival time constraints have been obtained using the observed radiation from gamma ray bursts [7], active galactic nuclei [8], and pulsars [9]. The best that can be done with the current data is $|\xi| \lesssim O(100)[7,8$, but more stringent limits can be expected in the future [10. Constraints of order $10^{-4}$ have been obtained on the difference in $\xi$ for different polarizations (birefringence) using spectropolarimetric observations of distant galaxies [11. Using the effective field theory result of [4] this yields the powerful new constraint $|\xi| \lesssim O\left(10^{-4}\right)$.

Complementary constraints were recently achieved by considering the threshold reactions of photon decay, vacuum Čerenkov radiation, and photon absorption involving high energy photons and electrons 13, 12, 14, 15, 16, 17, 19, 20. Energies around $10 \mathrm{TeV}$ are needed in order to put constraints of order unity on the deformation parameters for electrons and photons using threshold effects. Energies higher than this are achieved in astrophysical sources and hence threshold reactions place at least order unity bounds on $\eta, \xi$.

However, If we are to rule out quantum gravity effects characterized by (12) it is necessary to find observations that limit $\eta$ and $\xi$ to be much smaller 
than unity. None of the previous work has accomplished this for both electrons and photons. Here we show that the presence of synchrotron radiation from the Crab nebula, emitted by electrons cycling in a magnetic field, provides the new constraint $\eta>-7 \times 10^{-8}$ on the electron parameter $\eta$ within the effective field theory framework. This improves previous constraints by a very large amount. In particular it implies that the parameter $E_{Q G}$ of the popular phenomenological model of Ref. [6] (which corresponds to $M /|\eta|$, with $\xi=\eta$ in our notation) is bounded by $E_{Q G}>10^{7} M_{\text {Planck. }}$. (To be compatible with effective field theory that model must be modified to accommodate the polarization-dependence of the sign of $\xi$.) This is seven orders of magnitude stronger than the constraint from photon absorption $E_{Q G}>0.3 M_{\text {Planck }}$ [15, 16, 18] (see also [19, 20] for a debate on the validity of this constraint) and three orders of magnitude stronger than the new birefringence constraint of 4$]$.

Synchrotron radiation in the Crab nebula is produced by electrons cycling in a magnetic field of around $0.6 \mathrm{mG}$. To produce the observed radiation of energy $100 \mathrm{MeV}$ in this field requires in standard electrodynamics a gamma factor $\gamma=\left(1-v^{2}\right)^{-1 / 2}$ of $3 \times 10^{9}$, corresponding to an electron energy of $1500 \mathrm{TeV}$. To achieve this energy the electron velocity must differ from the speed of light by less than one part in $10^{19}$. Lorentz violation with negative $\eta$ puts an upper bound on the electron speed and hence an upper bound on the possible synchrotron radiation frequency. (The existence of a cutoff in the synchrotron radiation at high charged particle energies in the presence of Lorentz violation was previously noted in Ref. 21.) But to apply this reasoning we must first re-analyze the synchrotron emission process allowing for the Lorentz violating effects that we wish to bound.

In standard electrodynamics, accelerated electrons in a magnetic field emit synchrotron radiation with a spectrum that sharply cuts off at a frequency $\omega_{c}$ given by the formula [22]

$$
\omega_{c}^{\mathrm{LI}}=\frac{3}{2} \frac{e B \gamma^{2}}{m}
$$

where $B$ is the component of the magnetic field orthogonal to the electron path. The gamma factor $\gamma=E / m$ grows without bound with the electron energy $E$ so there is no upper limit on $\omega_{c}^{\mathrm{LI}}$.

Equation (3) is based on the electron trajectory in a given magnetic field, the radiation produced by a given current, and the relativistic relation between energy and velocity, all of which could be affected by Lorentz violation. 
We find that in the presence of Lorentz violation, (3) becomes

$$
\omega_{c}=\frac{3}{2} \frac{e B}{m} \frac{m \gamma(E)}{E} \gamma^{2}(E),
$$

where $\gamma(E)=\gamma(v(E)$ ). (For derivations of (4) and the constraint (15) below see the Methods section.) If $\eta$ is negative, electrons have a maximal velocity less than $c$, hence $\gamma(E)$ is a bounded function of $E$. This implies that there is a maximum achievable value of the cutoff frequency as given by equation (41), which we denote by $\omega_{c}^{\max }$. The value of the energy that yields this cutoff frequency is higher than the Lorentz-invariant value.

The rapid decrease in amplitude of synchrotron emission at frequencies larger than $\omega_{c}$ implies that most of the flux at a given frequency in a synchrotron spectrum is due to electrons whose $\omega_{c}$ is above that frequency. Thus $\omega_{c}^{\max }$ must be greater than the maximum observed synchrotron emission frequency $\omega_{\text {obs. }}$. This yields the constraint

$$
\eta>-\frac{M}{m}\left(\frac{0.34 e B}{m \omega_{\mathrm{obs}}}\right)^{3 / 2} .
$$

The strongest synchrotron constraint comes from the system that minimizes the ratio $B / \omega_{\text {obs }}$, which turns out to be the Crab nebula. The Crab nebula, a supernova remnant (SNR), is a bright source of radio, optical, Xray and gamma-ray emission, which exhibits a broad spectrum characterized by two marked humps. This spectrum is consistently explained by a combination of electron synchrotron emission and inverse Compton scattering of ambient photons by high energy electrons [23, 24]. In fact, no other model for the emission is under consideration, other than for producing some of the highest energy photons. For our constraint we assume that this standard SNR model is correct.

The Crab synchrotron emission has been observed to extend at least up to energies of about $100 \mathrm{MeV}$ 23, 24], just before the inverse Compton hump begins to contribute to the spectrum. The magnetic field in the emission region has been estimated by several methods which agree on a value between 0.15-0.6 mG (see e.g. [25] and references therein.) Two of these methods, radio synchrotron emission and equipartition of energy, are insensitive to Planck suppressed Lorentz violation, hence we are justified in adopting a value of this order for the purpose of constraining Lorentz violation. We shall use the largest value $0.6 \mathrm{mG}$ for $B$ since it yields the weakest constraint (Eq. (5) shows how the constraint scales with $B$ ). 
Using the above values for the magnetic field and the highest synchrotron frequency, Eq. (5i5) yields the lower bound

$$
\eta>-7 \times 10^{-8} \text {. }
$$

This corresponds to

$$
E_{\mathrm{QG}}=\frac{M}{|\eta|}>10^{26} \mathrm{GeV}
$$

in the phenomenological framework of Ref. [6]. Thus $E_{\mathrm{QG}}$ is constrained to be at least seven orders of magnitude larger than the Planck energy.

To complement the synchrotron constraint, which just bounds $\eta$ from below, one can use the vacuum birefringence and Cerenkov constraints. Lack of observed vacuum birefringence bounds the difference in $\xi$ for right and left circular polarized photons [11]. Using the effective field theory result of [4] that left and right circular polarized photons have opposite values for $\xi$, this yields the constraint $|\xi|<4 \times 10^{-4}$.

To bound $\eta$ from above we can use the vacuum Cerenkov constraint. As noted by previous authors [12, 13, 15, 17, modified dispersion can cause electrons to emit Čerenkov radiation in vacuum and rapidly lose energy. It was suggested in [18] that a very strong Cerenkov constraint is obtained for positive $\eta$ using the high energy electrons that produce the synchrotron radiation. But for positive $\eta$ the energy required to produce a given cutoff synchrotron frequency goes down, as the electron group velocity can increase beyond the speed of light and $\gamma(E)$ can diverge at finite energy. Hence the electron energy may be much lower than it is in the Lorentz-invariant case. (Nevertheless a constraint can be obtained in this way, as we shall show in a future publication with F. Stecker.) Thus we look to other high energy electrons whose existence is not in question.

The inverse Compton peak in the Crab spectrum contains energies up to $50 \mathrm{TeV}$. By energy conservation, this implies that electrons of at least 50 $\mathrm{TeV}$ propagate, hence the values of $\eta$ and $\xi$ must not allow these electrons to emit vacuum Čerenkov radiation. Within the region not ruled out by the birefringence bound, the results of [15, 16, 17] yield $\eta<0.01$.

Finally, recall that we neglected $\xi$ in obtaining the constraint. Using Eq. (13) one can see that this is justified everywhere in the region not already excluded by the absence of vacuum Cerenkov radiation. The worst case would be at the smallest $|\eta|$. With $\eta$ given by the lower bound ([6), Eq. (13) shows that $\xi$ can be neglected provided $|\xi| \lesssim 30$, which is a factor of nearly $10^{5}$ larger than the vacuum birefringence limit. 
Putting these observations together, we conclude that Lorentz violation suppressed by the ratio $E / E_{\text {Planck }}$ and compatible with effective field theory is constrained in all directions of the $\xi-\eta$ parameter space by much stronger than order unity bounds. The allowed region is a skinny rectangle, with bounds from vacuum birefringence above and below by $|\xi|<4 \times 10^{-4}$, from Crab synchrotron radiation on the left by $\eta>-7 \times 10^{-8}$, and from vacuum Cerenkov radiation on the right by $\eta<0.01$.

It is notable that, in such a short time after the recognition of the possibility of obtaining interesting bounds on Planck suppressed Lorentz violation, it has been so severely constrained. The constraint reported here on a possible maximal electron speed less than the speed of light is more than seven orders of magnitude better than previous limits. This limit is strong enough to conclude that quantum gravity scenarios postulating or implying this sort of Lorentz violation are not viable.

The type of Lorentz violation we have considered also violates CPT symmetry [4, the symmetry under combined charge conjugation, space inversion, and time reversal. The fact that it is ruled out might therefore be a consequence of CPT symmetry, rather than Lorentz symmetry. It thus makes sense to think seriously about constraining Lorentz violation suppressed by the second power, $\left(E / E_{\text {Planck }}\right)^{2}$. Some interesting bounds already exist at this order [26, 16], and other possibilities have been discussed [16, 27]. If it were eventually possible to achieve strong bounds at this order then it would be reasonable to conclude that Lorentz symmetry is simply not violated, since there is no other symmetry that could protect against this sort of Lorentz violation. Alternatively, if the search for such constraints finds a Lorentz violation, that would open up a much needed observational window on the difficult problem of quantum gravity.

\section{Methods}

To determine the effects of Lorentz violation of (3) we follow the heuristic derivation of (31) given in Ref. [22] assuming the framework of effective field theory. Without assuming Lorentz invariance, the purely kinematical result is

$$
\omega_{c}=\frac{3}{4} \frac{1}{R(E) \delta(E)} \frac{1}{c\left(\omega_{c}\right)-v(E)}
$$


where $R(E)$ is the radius of curvature of the orbit, $\delta(E)$ is the opening angle for the forward-directed radiation pattern, and $c\left(\omega_{c}\right)$ and $v(E)$ are the group velocities of the radiation and electron respectively. The solution of Eq. (8) for $\omega_{c}(E)$ determines the cutoff synchrotron frequency. In Eq. (8) we have used the fact that the electron and photon speeds are very close to the low energy speed of light $c$, which is set equal to unity. The numerical constant is chosen to yield the correct relativistic result (3) with $\delta(E)=\gamma^{-1}(E)$ (where $\gamma(E)=\gamma(v(E)))$.

The radius $R(E)$ for a given energy is determined by the equation of motion of the electron in a magnetic field. All Lorentz violating terms in the equation of motion are suppressed by $M^{-1}$, and the leading high energy corrections come from modifications to the minimal coupling terms. To estimate the magnitude of the change we use the dispersion relation as the Hamiltonian. As usual the minimal coupling is incorporated replacing the momentum by $\mathbf{p}-e \mathbf{A}$, where $\mathbf{A}$ is a vector potential for the magnetic field. This yields the equation of motion $\mathbf{a}=[1+3 \eta E / 2 M](e / E) \mathbf{v} \times \mathbf{B}$, where we have kept only the lowest order term in $\eta$ and assumed relativistic energy $E \gg m$. Since $E \ll M$, the presence of the Lorentz violation makes very little difference to the orbital equation, hence we conclude that to a very good approximation the radius is related to the magnetic field and the energy of the electron by the standard formula $R(E)=E / e B$ (where again the speed of the electron has been set equal to unity).

The angle $\delta(E)$ scales in the Lorentz invariant case as $\gamma^{-1}(E)$. Since $R(E)$ and hence the charge current is nearly unaffected by the Lorentz violation, any significant deviation in $\delta(E)$ can only come from the modified response of the electromagnetic field to a given current. The amplitude of the modification is suppressed by at least one power of $M$ however, so dimensional analysis implies that it is suppressed by something of order $\xi \omega / M$. For the Crab synchrotron radiation used in our constraint $\omega$ is $100 \mathrm{MeV}$, which yields a suppression factor of $10^{-20} \xi$. Therefore the amplitude of the electromagnetic field modification is negligible in comparison to the zeroth order synchrotron radiation (which is still present), and hence $\delta(E)$ scales with $\gamma^{-1}(E)$ in the usual way.

Since the emitted photons have relatively low energy compared to the electrons, it turns out that $\xi$ can be neglected in the relevant region of parameter space. Thus, since $v(E)$ is very close to $c$, the reciprocal of the difference of group velocities in the last term of Eq. (8) is well approximated by $2 \gamma^{2}(E)$. This yields (4). 
The maximum synchrotron frequency $\omega_{c}^{\max }$ is obtained by maximizing $\omega_{c}$ (4) with respect to the electron energy, which amounts to maximizing $\gamma^{3}(E) / E$. The difference of group velocities is given by

$$
c(\omega)-v(E)=\xi \frac{\omega}{M}+\frac{m^{2}}{2 E^{2}}-\eta \frac{E}{M},
$$

where we have used the dispersion relations (112) and dropped higher order terms. Dropping the $\xi$ term this yields

$$
\gamma(E) \approx\left(\frac{m^{2}}{E^{2}}-2 \eta \frac{E}{M}\right)^{-1 / 2},
$$

Using this expression we find

$$
\omega_{c}^{\max }=0.34 \frac{e B}{m}(-\eta m / M)^{-2 / 3},
$$

which is attained at the energy $E_{\max }=\left(-2 m^{2} M / 5 \eta\right)^{1 / 3}=10(-\eta)^{-1 / 3} \mathrm{TeV}$. Since $\eta$ is negative this is higher than the Lorentz invariant value that produces the same frequency, but only by a factor of order unity that works out to be $(9 / 5)^{3 / 4} \approx 1.55$. The energy of the electrons that produce the synchrotron radiation of frequency $100 \mathrm{MeV}$ is $1500 \mathrm{TeV}$ in the Lorentz invariant case. If this were the maximum frequency in the Lorentz violating case, it would be produced by $\sim 2300 \mathrm{TeV}$ electrons.

We now analyze the question of neglecting $\xi$ in evaluating the difference of group velocities (91). At the energy $E_{\max }$, the ratio of the $\xi$-dependent term to the other terms is given by

$$
\frac{|\xi| \omega}{\left(m^{2} M / 2 E^{2}\right)-\eta E}=3 \times 10^{-11}|\xi|(-\eta)^{-4 / 3} .
$$

Hence neglecting $\xi$ is justified provided

$$
|\xi| \lesssim 10^{11}(-\eta)^{4 / 3}
$$

\section{Acknowledgements}

We wish to thank F.A. Aharonian, G.E. Allen, G. Amelino-Camelia, and F. Stecker for helpful discussions. This work was supported in part by the NSF. 


\section{References}

[1] Gambini, R. \& Pullin, J. Nonstandard optics from quantum spacetime. Phys. Rev. D 59, 124021 (1999).

[2] Carroll, S. M., Harvey, J. A., Kostelecky V. A., Lane, C. D., \& Okamoto, T. Noncommutative field theory and Lorentz violation, Phys. Rev. Lett. 87, 141601 (2001).

[3] Burgess, C. P., Cline, J., Filotas, E., Matias, J. \& Moore, G. D. Loopgenerated bounds on changes to the graviton dispersion relation. JHEP 0203, 043 (2002).

[4] Myers R. C. \& Pospelov, M. Experimental challenges for quantum gravity. Phys. Rev. Lett. 90, 211601 (2003).

[5] Pavlopoulos, T. G. Breakdown of Lorentz invariance, Phys. Rev. 159, 1106-1110 (1967).

[6] Amelino-Camelia, G., Ellis, J. R., Mavromatos, N. E., Nanopoulos, D. V. \& Sarkar, S. Tests of quantum gravity from observations of gamma-ray bursts. Nature 393, 763-765 (1998).

[7] Schaefer, B. Severe limits on variations of the speed of light with frequency. Phys. Rev. Lett. 82, 4964-4966 (1999).

[8] Biller, S. D. et al. Limits to quantum gravity effects from observations of TeV flares in active galaxies. Phys. Rev. Lett. 83, 2108-2111 (1999)

[9] Kaaret, P. Pulsar radiation and quantum gravity. Astron. Astrophys. 345, L32-L34 (1999).

[10] Norris, J. P., Bonnell, J. T., Marani G. F. \& Scargle, J. D. GLAST, GRBs, and quantum gravity. Bulletin of the American Astronomical Society, Vol. 31, p.717.

[11] Gleiser R. J. \& Kozameh, C. N. Astrophysical limits on quantum gravity motivated birefringence. Phys. Rev. D 64, 083007 (2001).

[12] Gonzalez-Mestres, L. Cosmological implications of a possible class of particles able to travel faster than light. Nucl. Phys. Proc. Suppl. 48, 131-134 (1996). 
[13] Coleman, S. \& Glashow, S. L. High-energy tests of Lorentz invariance. Phys. Rev. 59, 116008, (1999).

[14] Stecker, F. W. \& Glashow, S. L. New tests of Lorentz invariance following from observations of the highest energy cosmic gamma rays. Astropart. Phys. 16, 97-99 (2001).

[15] Jacobson, T., Liberati, S. \& Mattingly, D. TeV astrophysics constraints on Planck scale Lorentz violation. Phys. Rev. D 66, 081302 (2002).

[16] Jacobson, T., Liberati, S. \& Mattingly, D. Threshold effects and Planck scale Lorentz violation: Combined constraints from high energy astrophysics. Phys. Rev. D 67, 124011 (2003).

[17] Konopka T. J. \& Major, S. A. Observational limits on quantum geometry effects. New J. Phys. 4, 57.1-8 (2002).

[18] Stecker, F. W. Tests of quantum gravity and large extra dimensions models using high energy gamma ray observations. arXiv:astro-ph/0304527.

[19] Amelino-Camelia, G. Improved limit on quantum-spacetime modifications of Lorentz symmetry from observations of gamma-ray blazars. arXiv:gr-qc/0212002.

[20] Jacobson, T., Liberati S. \& Mattingly, D. Comments on 'Improved limit on quantum-spacetime modifications of Lorentz symmetry from observations of gamma-ray blazars'. arXiv:gr-qc/0303001.

[21] Gonzalez-Mestres, L. Lorentz symmetry violation and acceleration in relativistic shocks. AIP Conf. Proc. 558, 874-877 (2001).

[22] Jackson, J. D. Classical Electrodynamics, 3rd edn, 671 (Wiley \& Sons, New York, 1998).

[23] Atoyan A. M. \& Aharonian, F.A. On the mechanisms of gamma radiation in the Crab Nebula. Mon. Not. R. Astron. Soc. 278, 525-541 (1996).

[24] de Jager, O. C. et. al.. Gamma-Ray Observations of the Crab Nebula: A Study of the Synchro-Compton Spectrum. Astrophysical J. 457, 253-266 (1986). 
[25] Hillas A. H. et al. The Spectrum of TeV gamma rays from the crab nebula. Astrophysical J. 503, 744-759 (1998).

[26] Aloisio, R., Blasi, P., Ghia P. L. \& Grillo, A. F. Probing the structure of space-time with cosmic rays. Phys. Rev. D 62, 053010 (2000).

[27] Amelino-Camelia, G. Proposal of a second generation of quantumgravity-motivated Lorentz-symmetry tests: Sensitivity to effects suppressed quadratically by the Planck scale. arXiv:gr-qc/0305057. 